\title{
Wie das Moosglöckchen zu seinem Namen kam - Geschichten und Gedanken zum 300. Geburtstag von CarL von Linné, Teil 1
}

\author{
Karin STEINeCKe
}

\begin{abstract}
In 2007 not only botanists are celebrating the tercentenary of the birth of the famous swedish naturalist CARL VON LINNÉ. The following article describes the life of LinNÉ, starting with telling the story how the little twinflower got its scientific name Linnaea borealis.

\section{Zusammenfassung}

Im Jahr 2007 feiern nicht nur Botaniker den 300. Geburtstag des berühmten schwedischen Naturforschers CARL vON LinNÉ. Im folgenden Beitrag werden Leben und Werk LinNés vorgestellt. Ergänzend wird berichtet, wie das Moosglöckchen zu seinem wissenschaftlichen Namen Linnaea borealis gekommen ist.
\end{abstract}

\section{Einleitung}

Wer das kleine Moosglöckchen schon einmal am Waldboden wachsen gesehen hat, ist begeistert. Die winzigen rosafarbenen Blütenglöckchen dieser kriechenden Pflanze hängen so anmutig und gleichzeitig erhaben immer zu zweien auf langen dünnen Stängeln, dass man beinahe ein leises Klingen der Glöckchen im Halbdunkel des Nadelwaldes zu hören vermag. Dazu verströmen sie einen süßen, mandel- oder fast vanilleartigen Duft, den man dieser zierlichen Waldbodenpflanze gar nicht zutrauen möchte und der ihr blumenelfenhaftes Wesen noch unterstützt (Abb. 1).

Doch wie unscheinbar und zart dieses Pflänzchen auch sein mag, es erinnert uns mit seinem wissenschaftlichen Namen Linnaea borealis an einen großen schwedischen Naturforscher, Arzt und Botaniker, der mit seinen Forschungen und Ideen die naturwissenschaftliche Erkenntnisbildung entschieden vorangetrieben hat. Die Rede ist von CARL v. LINNÉ, dessen Geburtstag sich im Jahr 2007 zum 300. Mal jährt. Der nachfolgende Artikel erhebt keinen Anspruch auf eine vollständige Biographie Linnés. Ausführliche Linné-Lebensläufe kann man anlässlich des diesjährigen LinNÉ-Jahres vor allem im Internet gleich mehrere finden. Es sollen hier vielleicht eher unbekannte Details aus LinNÉs Leben herausgegriffen werden. $\mathrm{Zu}$

Abb. 1: Die Moosglöckchen-Elfe, Originalzeichnung von G. Herzog.
Beginn werden seine Lieblingspflanze, das kleine Moosglöckchen, sowie die schwedische Landschaft, in der LINNÉ aufgewachsen ist, vorgestellt. Wichtigste Quellen für die biographischen Informationen über LinNé sind GOERKE (1989) sowie die anlässlich des LINNÉ-Jahres von der Universität Uppsala erstellte Internetseite „LINNÉ online“.

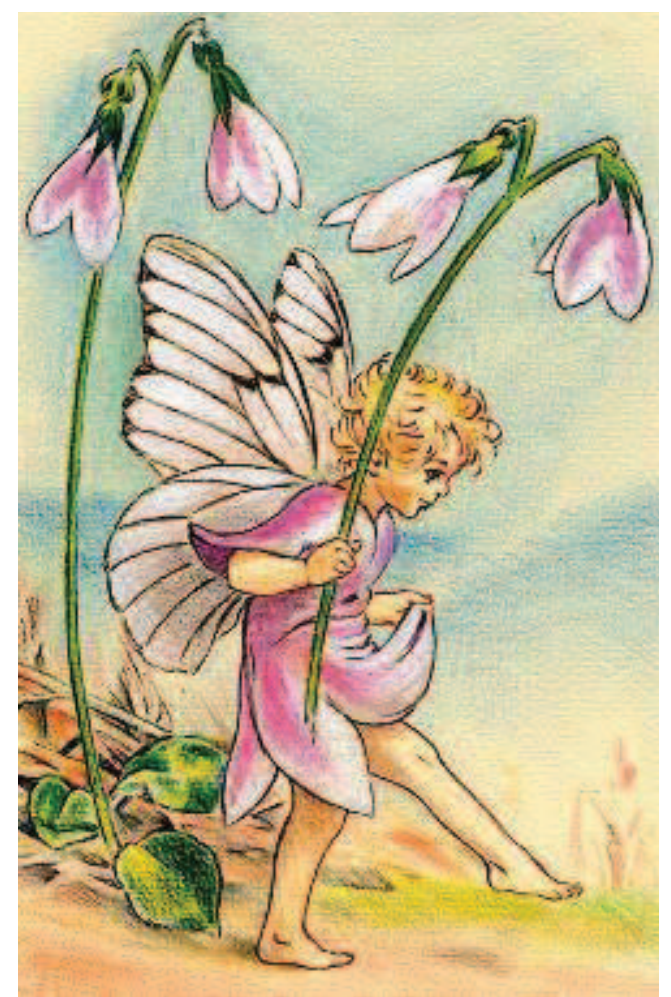




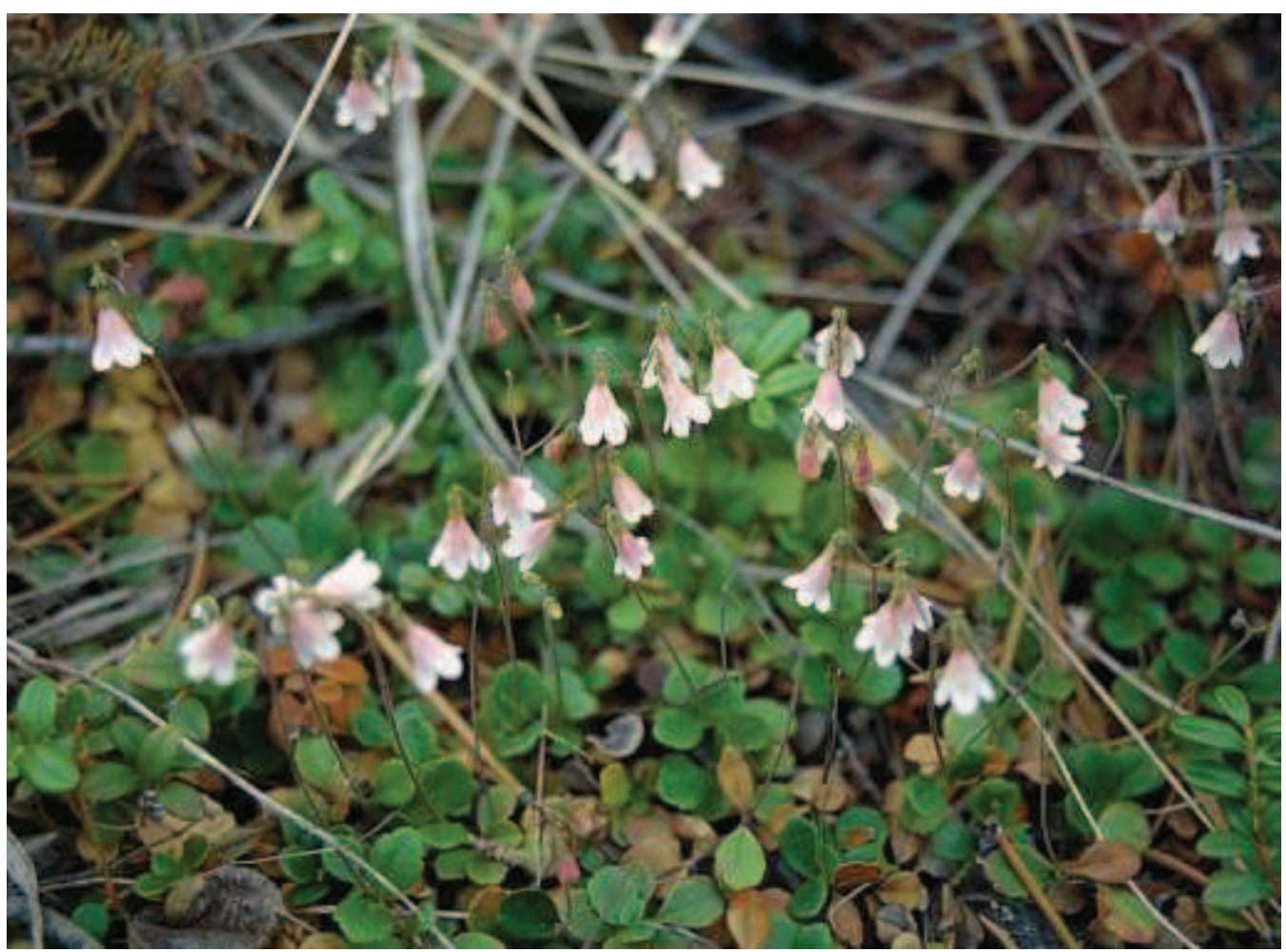

\section{Småland, Linnés Heimat}

LinNÉs Heimat ist der Teil Schwedens, den die berühmte Kinderbuchautorin Astrid LindGREN durch ihre Erzählungen bekannt gemacht hat. Die Rede ist von der südschwedischen Provinz Småland, die sich nördlich von Schonen und Blekinge in einem breiten Streifen entlang der Ostseeküste bis zum Vätternsee erstreckt. Und wer die Bilder der Landschaft um Bullerbü und Katthult vor Augen hat, kann sich vorstellen, wie es in der Umgebung des Hofes Råshult, dem Geburtsort von LinNÉ, früher aussah und zum großen Teil auch heute noch aussieht. Die Landschaft Smålands ist durch die Eiszeit geprägt. Weite Bereiche sind mit ausgedehnten Nadel- und Birkenmischwäldern bestanden, in die zahlreiche Seen und Moore eingebettet sind. Findlinge und Rundhöcker sind markante Zeugen der eiszeitlichen Vergletscherung. Småland wurde in historischen Zeiten aber auch sehr intensiv landwirtschaftlich genutzt, so dass für die småländische Landschaft ebenso die Viehweiden mit den schönen Weidegattern und die kleinen, häufig rot gestrichenen Bauernhäuser typisch sind.

LinNé wuchs also inmitten der pflanzengeographisch interessanten Übergangszone zwischen temperatem und borealem Waldgürtel auf. Dieser gilt als besonders artenreich und botanisch attraktiv, da sich die entsprechenden Geoelemente hier vermischen. So finden sich beispielsweise in der Strauch-, Kraut- und Moosschicht nebeneinander anspruchsvolle Arten der südlichen Buchenwälder wie die Frühlingsplatterbse (Lathyrus vernus) oder das Buschwindröschen (Anemone sylvestris) und weniger anspruchsvolle boreale Arten wie das Moosglöckchen (Linnaea borealis) oder verschiedene Vaccinium-Sippen (GLÄsSER et al. 2003). Wen wundert es also, dass Linné sich in seiner Kindheit und Jugend an der Pflanzenwelt der Umgebung erfreute und sich später mit ihrer Klassifizierung beschäftigte.

Abb. 2: Linnaea borealis im Lebensraum, Yellowknife, Kanada. 
3. Das Moosglöckchen (Linnaea borealis), die Lieblings- und Wappenblume Linnés Das Moos- oder Erdglöckchen (Linnaea borealis, Abb. 2-4) ist in Skandinavien weit verbreitet (Mossberg et al. 1992). Es ist eine circumpolar vorkommende arktisch-alpine Art, die typisch für die Alpen sowie die gesamte boreale Zone der Nordhalbkugel ist (Abb. 5). Es werden die drei Unterarten subsp. borealis (Eurasien), susp. americana (Nordamerika) und subsp. longiflora (Kordilleren Nordamerikas) unterschieden. In Deutschland kommt Linnaea borealis indigen u. a. in den Allgäuer Alpen vor, es sind aber auch neophytische Vorkommen aus Hamburg, Schleswig-Holstein, Niedersachsen und Mecklenburg-Vorpommern bekannt (KoRNECK et al. 1996). Natürlicher Lebensraum sind schattige bis halbschattige und feuchte bis trockene, aber moosige Waldbodenstandorte in lichten Nadelund Mischwäldern. Dort wächst es als kriechender Halbstrauch mit bis 1,20 m langen, dünnen Sprossen. Die immergrünen Blätter sind gegenständig, rundlich bis eiförmig und nur schwach gestielt. Die zartrosafarbenen bis weißen, nur etwa 5-8 mm langen Blüten stehen paarweise an aufrechten Stängeln (daher der englische Name twinflower $=$ Zwillingsblume), sind glockenförmig mit fünf Kronzipfeln. Die Blütezeit ist von Juni bis September. Die einzelnen Blüten halten sich über mehrere Tage, die Früchte sind einsamige, trockene und stachelige Kapseln (Abb. 4).

Linnaea borealis wurde lange Zeit u.a. mit Sambucus, Viburnum (beide heute Adoxaceae), Lonicera und Symphoricarpos den Caprifoliaceae zugeordnet. Nach neuen molekularphylogenetischen Befunden wird Linnaea zusammen mit Abelia, Dipelta, Kolkwitzia und Zabelia zur Familie der Linnaeaceae gestellt. Das Moosglöckchen ist einzige Art der Gattung Linnaea. Während alle Arten der nahe verwandten Gattungen echte Sträucher oder Lianen sind (Abb. 6), ist das Moosglöckchen in diesem Verwandtschaftskreis der einzige nur schwach verholzte Halbstrauch von maximal $15 \mathrm{~cm}$ Höhe.

Abb. 3: „Zwillingsblüte“ des Moosglöckchens, aufgenommen im Oberengadin, Schweiz.

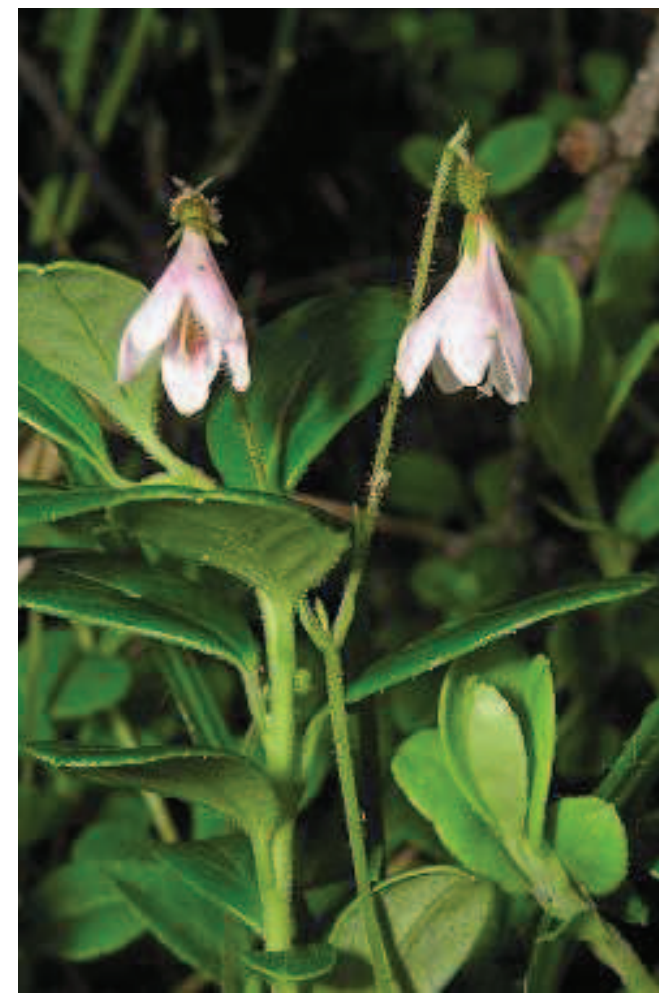

Schon hier sei erwähnt, dass das Moosglöckchen zeitlebens die Lieblingspflanze LinNÉs war; er kannte diese Art bereits von seiner Kindheit an aus den Wäldern seines Heimatortes. Gern ließ er sich mit Linnaea borealis in der Hand oder am Hut portraitieren (vgl. Abb. 8), später arbeitete er das Abbild dieser attraktiven Pflanze auch in sein Familien- bzw. Adelswappen ein (s.u.). Heute gilt das Moosglöckchen auch als die regionale Landschaftsblume Smålands. Zudem schmücken viele, insbesondere nordische Künstler und Designer ihre Werke (u. a. Silberschmuck, Porzellan, Heimtextilien, Briefmarken) mit Motiven dieser hübschen Pflanze (Abb. 7).

\section{LiNNÉs Leben \\ 4.1 Familie und Familienname}

LiNNÉ entstammt mütterlicherseits einer eingesessenen protestantischen Pastorenfamilie in Südschweden. Vater, Großvater und Urgroßvater seiner Mutter, der Christina Linnaeus (geb. Broderson, 1688-1733), waren Pfarrer 


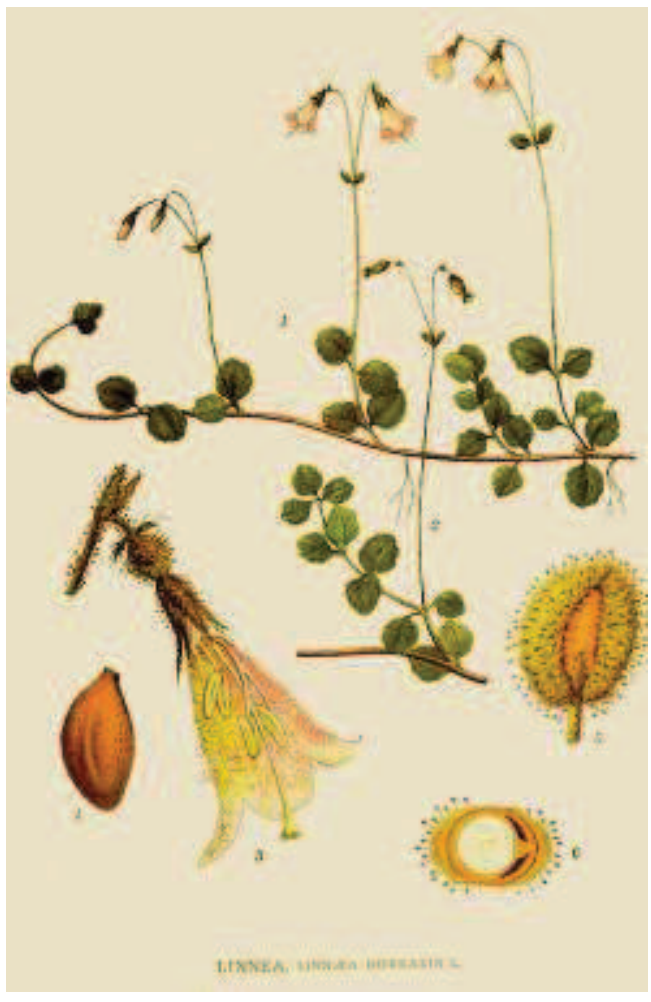

im Gemeindebezirk Stenbrohult südlich von Växjö in Småland. Auch die Mutter von CHrIsTINA BRODERSON wuchs in einer Pfarrersfamilie aus dem benachbarten Visseltofta auf. Hier hatte sich Jörgen Simonsson ScheE (16121692) als Pfarrer niedergelassen, nachdem er als Waisenjunge nach der Hexenverbrennung seiner Mutter in Stavanger aus Norwegen geflohen und schließlich Geistlicher geworden war. LINNÉs Vater, Nils (Nicolaus) Ingemarsson LinNaeus (1674-1748) hingegen stammte aus einer bäuerlichen Familie aus Vittaryd etwa $50 \mathrm{~km}$ von Stenbrohult entfernt. Diese Gegend galt als eine der ärmsten Landstriche Smålands. Dennoch studierte der Bauernsohn NiLs IngEMARsson wie schon zuvor einige Verwandte seiner Eltern Theologie an der Universität in Lund. Beim Eintritt in den Gelehrtenstand war es damals üblich, sich zusätzlich zu dem Vaternamen einen besonderen und festen Beinamen zuzulegen, der auch weiter vererbt wurde. Während hierfür häufig Ortsnamen ausgewählt wurden, griff der botanisch sehr interessierte NiLs eine
Idee auf, die seine bereits erwähnten Onkel mütterlicherseits bei der Namensfindung im Zuge der Aufnahme des Theologiestudiums gehabt hatten. Diese hatten sich nämlich nach einer mächtigen uralten dreistämmigen Sommerlinde (Tilia cordata) in der Nähe des väterlichen Bauernhofes Jonsboda Linnegård in Vittaryd, die auch heute noch - wenn auch in einem sehr schlechten Zustand - existieren soll, die Namen Lindelius (nach dem schwedischen Wort für Linde $=$ lind) und Tiliander (nach dem lateinischen Wort für Linde = tilia) gegeben. Linnés Vater ehrte diesen Familienbaum schließlich durch den Zunamen LinNaEus, was als eine Latinisierung des småländischen Dialektwortes linn für Linde zu verstehen ist. Somit zeugt bereits der Familienname LinNés von der großen Begeisterung der engeren Familie für Pflanzen.

Insbesondere der Onkel Sven Tiliander (1637-1710) hatte großen Einfluss auf die botanischen Neigungen von LINNÉs Vater. Sven Tiliander arbeitete von 1667 bis 1673 als Hausprediger des schwedischen Generalgouverneurs Henrik Horn in Bremen, wo er die üppigen und gepflegten Bürgergärten dieser Hansestadt kennen lernte. Wieder zurück in Schweden inspirierten sie ihn wohl zur Anlage eines mit seltenen Blumen und Pflanzen ausgestatteten Pfarrgartens, der wiederum seinen Neffen NILs stark beeindruckte. Nach Beendigung seines Theologiestudiums bekam NiLs Linnaeus 1703 vom Pfarrer in Stenbrohult die vakante Stelle eines Kaplans angeboten. In der Gemeinde erfreute sich NiLs großer Beliebtheit und wurde alsbald zum Hilfsprediger ernannt. Er bezog den kleinen Hof Råshult, und legte dort - dem Vorbild seines Onkels Sven TilianDER folgend - einen blumenreichen Pfarrgarten an. Zudem fand er Gefallen an der Pfarrerstochter Christina, die er 1706 heiratete. Linné wurde als erstes Kind der Familie am 13. Mai 1707 geboren. Dieses Datum entspricht dem 23. Mai nach dem heute gültigen Gregoriani-

Abb. 4: Linnaea borealis in einer gut 100 Jahre alten Zeichnung von Carl Axel Magnus Lindman. 


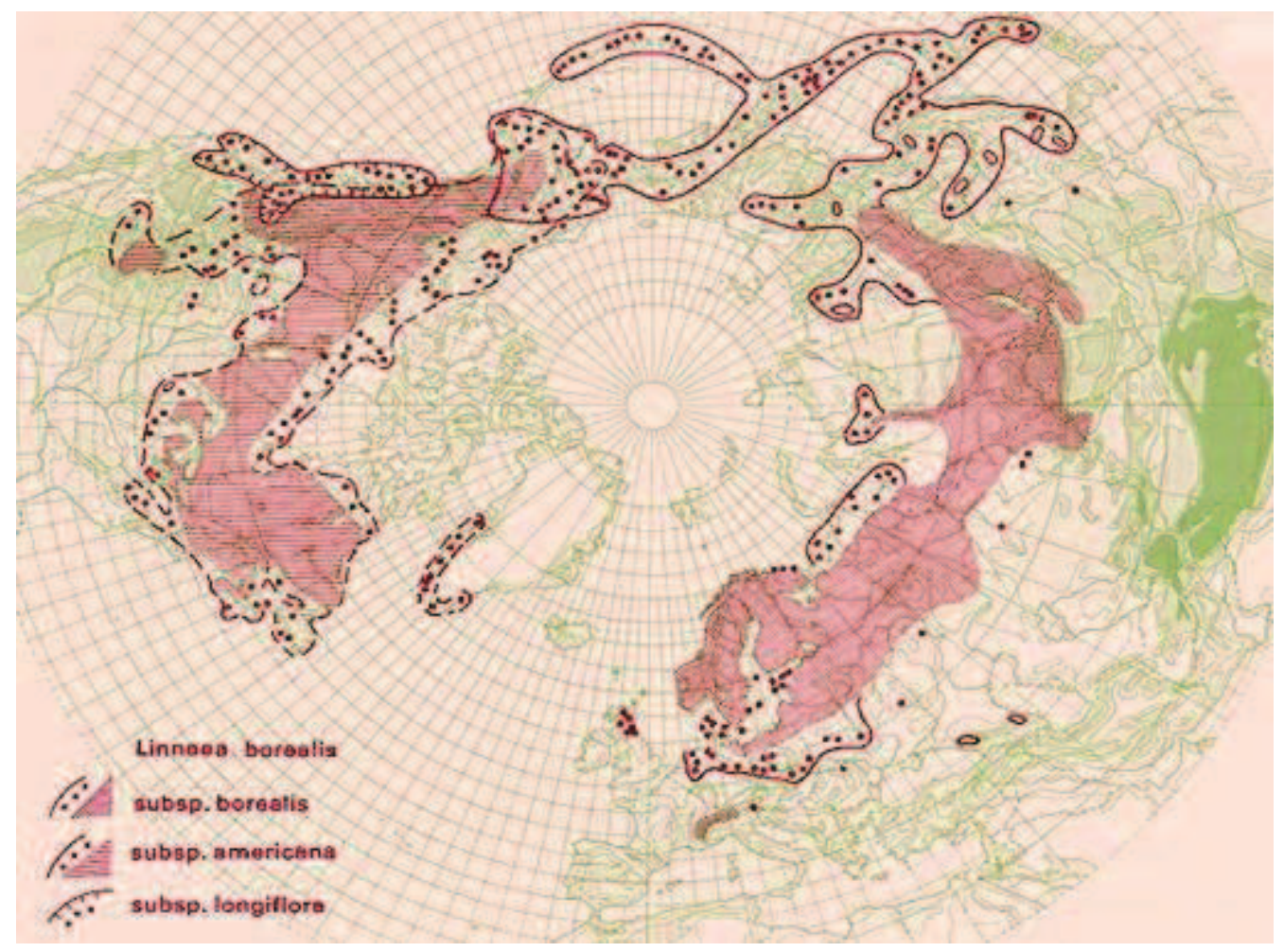

schen Kalender, der in Schweden erst im Jahr 1753 eingeführt wurde. Seinen Vornamen Carl bzw. Carolus erhielt der Sohn nach dem damals regierenden erfolgreichen König von Schweden (KARL XII, 1686-1718). Erst sehr viel später, bei der Erhebung in den Adelsstand, wurde sein Name von Carolus Linnaeus in Carl v. Linné abgewandelt (s. u.).

\subsection{Kindheit und Jugend}

Nach dem Tod des Schwiegervaters im Jahre 1709 wurde Nils Linnaeus neuer Gemeindepfarrer von Stenbrohult und siedelte mit seiner Familie ins dortige Pfarrhaus um, wo er trotz seines geringen Vermögens gleichfalls einen schönen Pfarrgarten mit seltenen Stauden und Gehölzen anlegte. In Stenbrohult wurden auch seine weiteren Kinder Anna Maria Linnaeus

Abb. 5: Verbreitungskarte von Linnaea borealis, verändert nach http://www.ubcbotanicalgarden.org/potd/2005/06/ linnaea_boreali.php (University of British Columbia, 2004)
(1710-1769), Sophia Juliana Linnaeus (1714-1771), SAMUEl LinNaEus (1718-1797) und Emerentia Linnaeus (1723-1753) geboren. Seinem ältesten Sohn CARL brachte er im Pfarrgarten und bei gemeinsamen Spaziergängen durch die småländische Natur schon früh die Pflanzenkunde näher. Daneben unterrichtete er ihn aber auch im Lesen und Schreiben, wie es sich früher für einen Pfarrershaushalt gehörte. CARL begeisterte sich sehr für die Natur und die Pflanzenwelt, dennoch sahen die Eltern für ihn in Wahrung der Familientradition eine Laufbahn als Geistlicher vor. Je älter CARL wurde, um so schwerer fiel ihm aber die Teilnahme am Schulunterricht, der ihm ab dem 7. Lebensjahr überwiegend durch Hauslehrer und an der Grundschule in Växjö zuteil wurde. Viel lieber hielt er sich draußen in der Natur auf, so dass seine Lehrer und Eltern an ihm und seinen schlechten Noten fast verzweifelten. Mit 17 Jahren kam CARL LinnaEus schließlich auf das Gymnasium in Växjö. Auch hier fiel er seiner Neigung entsprechend durch gute Noten in den 


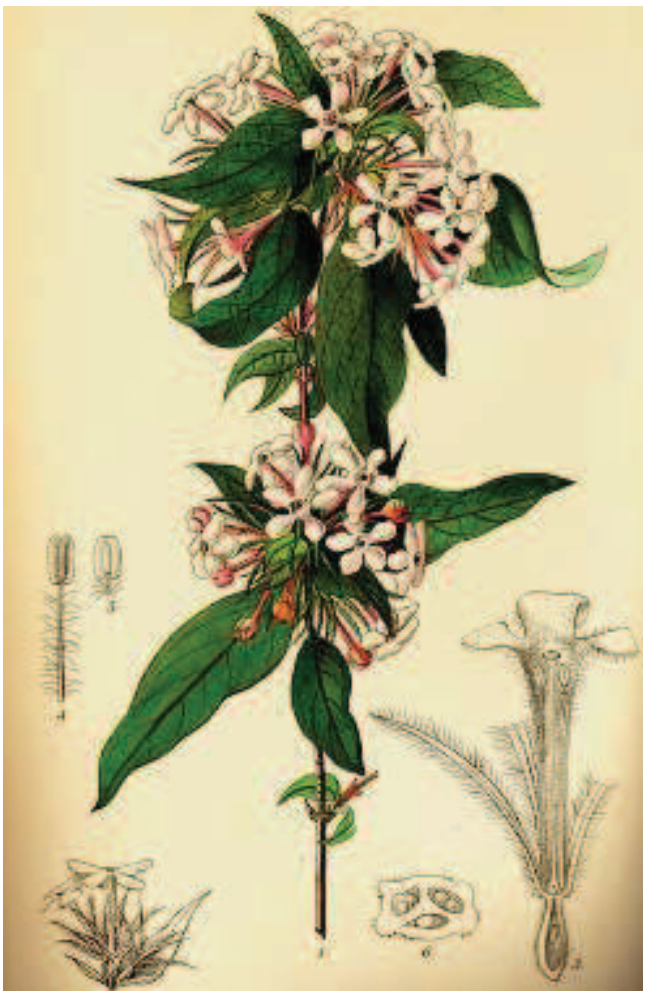

naturwissenschaftlichen Fächern und durch schlechteste Beurteilungen in Fächern wie Theologie, Moral, Griechisch oder Hebräisch auf. Dennoch hielten die Eltern an ihrem Wunsch fest, ihren Sohn zu einem Geistlichen zu machen. Nur dem Arzt und Lehrer der Naturwissenschaften am Gymnasium in Växjö, Dr. Johan Stensson Rothman (1684-1762), ist es zu verdanken, dass LINNÉ nicht zum Theologiestudium gezwungen wurde oder - wie andere Lehrer angeraten hatten - die Schulausbildung abbrach und statt eines Studiums eine Handwerkerausbildung begann. RothMANN erkannte die außergewöhnlichen naturwissenschaftlichen Fähigkeiten LiNNÉs und bat den Vater in einer Unterredung, ihn noch ein Jahr weiter zur Schule zu schicken und ihn auf ein Medizinstudium vorbereiten zu dürfen. LiNNÉs Vater ging auf diesen Wunsch ein; im Gegenzug förderte Rothman im folgenden Jahr Linné in vielfältiger Weise und erwirkte so einen erfolgreichen Abschluss am Gymnasium. Immer wieder ist aber zu lesen, dass der Vater LinNÉs
Wunsch nach einem Medizinstudium und seine Absprachen mit dem Lehrer lange Zeit vor der Mutter geheim hielt und diese erst zu Beginn des Studium von seiner definitiven Abkehr der geistlichen Laufbahn erfuhr. Diese schlug jedoch Linnés jüngerer Bruder SAmuel ein, der später Pfarrer in Stenbrohult wurde.

\subsection{Studium in Lund und Uppsala}

Im Sommer 1727 begann der nun mittlerweile 20-jährige LinNé an der Universität Lund, an der auch sein Vater studiert hatte, mit großem Eifer sein Medizinstudium, das in der damaligen Zeit einem kompletten Studium der Naturwissenschaften entsprach. Einen großen Teil nahmen dabei auch die botanischen Vorlesungen und Seminare ein. LiNNÉ ging auf zahlreiche Exkursionen in die Umgebung von Lund und vervollständigte dabei seine Herbarien und Sammlungen. Während er seinen eigenen Angaben zufolge vom viel beschäftigten und oft nicht verfügbaren Lehrstuhlinhaber der medizinischen Fakultät in Lund, JонаN Јаков DӧвELIUS, nur wenig Neues lernen konnte, profitierte LINNÉ während seines einen Studienjahres in Lund insbesondere durch seinen Aufenthalt im Hause des Historikers, Arztes und Dozenten Dr. Kilian Stobaeus (1690-1742). Dieser machte Linné seine umfangreichen Pflanzen-, Tier- und Mineraliensammlungen sowie seine gut ausgestattete Bibliothek für Studien zugänglich und umsorgte den armen Studenten finanziell und ideell wie einen Sohn. Diese Beziehung entwickelte sich angeblich, nachdem LINNÉ sich heimlich aus der Bibliothek STOBAEus botanische Fachbücher entliehen hatte, um diese nachts in seiner Kammer unter der Bettdecke bei Kerzenschein zu lesen. Die Haushälterin bemerkte dies und meldete den Vorfall aus Angst vor einem Brand dem Hausherrn. STOBAEUs jedoch war darüber nicht erbost, sondern erfreute sich zutiefst an der naturwissenschaft-

Abb. 6: Abelia triflora aus Curtis's Botanical Magazine, Nr. 9131; die Gattung Abelia ist eine der nächsten Verwandten des Moosglöckchens. 
lichen Begeisterung Linnés. Trotz dieser väterlichen Verbundenheit, die zeitlebens bestehen blieb, wechselte LINNÉ bereits nach einem Jahr den Studienort und zog nach Uppsala. Grund für diesen Wechsel war der erneute Rat seines ehemaligen Lehrers Rothman.

LinNé setzte sein Studium im Herbst 1728 an der Universität in Uppsala fort. Zunächst war er jedoch von den Studienbedingungen in Uppsala wenig begeistert. Die medizinische Fakultät war klein und unvollständig besetzt und wurde im wesentlichen von LARS ROBERG (1664-1742) und Olof Rudbeck dem Jüngeren (1660-1740) geführt, dessen Vorlesungen über Anatomie und Botanik Linné jedoch aufmerksam verfolgte. Der große Botanische Garten schien verwahrlost, zog LINNÉ aber magisch an. Als sein Studiengeld zur Neige ging, fand LINNÉ glücklicherweise auch in Uppsala wieder einen Gönner: den Theologen, Botaniker und Physiker Olof Celsius der Ältere (16701756), der den wissbegierigen LiNNÉ für einige Monate in seinem Hause aufnahm. Auch Olof RudBeck war LinNé zugetan, ließ seine jüngsten Söhne - er hatte 24 Kinder (!) - von ihm in seinem Hause unterrichten und sorgte schließlich dafür, dass LinNé bereits 1730 als Student im dritten Studienjahr eigene Lehrveranstaltungen der Botanik halten durfte und die Aufsicht über den Botanischen Garten übertragen bekam. Linné hatte nämlich zu Beginn des Jahres 1730 mit seiner ersten kleinen Schrift „Preludia Sponsalarium Plantarum “, in der er die Sexualität und die Fortpflanzungsorgane der Pflanzen beschrieb, großes Aufsehen erregt. Auch einige weitere wichtige Manuskripte, die später als „Bibliotheca Botanica “(1736), „Critica Botanica “(1737), „Genera Plantarum“ (1737) oder „Classes Plantarum“ (1738) veröffentlicht und berühmt wurden, begann LINNÉ bereits während seiner fruchtbaren Zeit im Hause RudBecks. Hier bekam er schließlich wohl auch den Anstoß zu seiner Lappland-Expedition

Abb. 7: Porzellan-Schale mit Linnaea-Motiven aus der Service-Serie Botanica von Villeroy \& Boch auf einem schwedischen Leinendeckchen der Firma Fröso Handtryck AB.

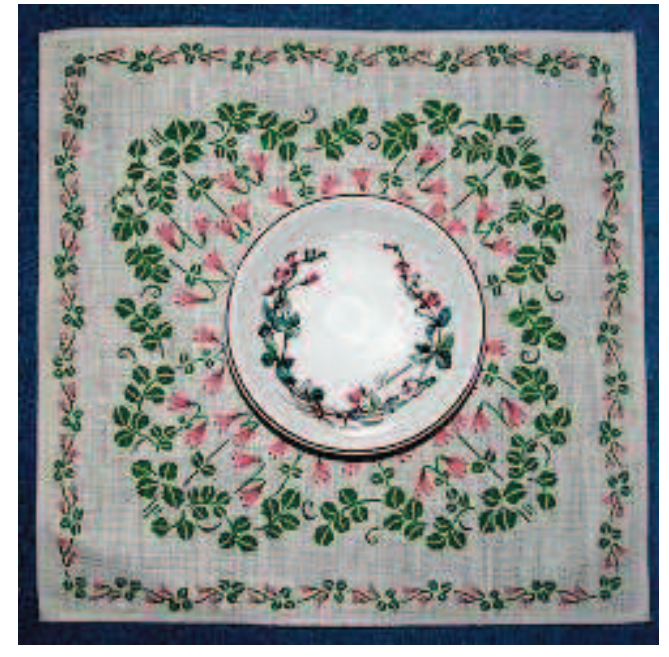

im Jahr 1732, da RudBeck selbst in jungen Jahren durch die nördlichste und noch weitgehend unerforschte Provinz Schwedens gereist war und davon schwärmte. Da die Akademie der Wissenschaften in Uppsala auf königliches Geheiß gerade mit der Durchführung einer Forschungsreise nach Lappland zur Erkundung wirtschaftlicher Erschließungsmöglichkeiten des Nordens beauftragt worden war, bewarb sich LINNÉ um diese attraktive Aufgabe und erhielt auch kurz darauf Zuschlag und Reisemittel zur Durchführung dieser Expedition, auf der er erhebliche Beiträge zur naturkundlichen Erforschung Lapplands leistete. Publikationen die sich aus dieser Reise entwickelten, waren die „Flora Lapponica“ (1737) sowie ein erst sehr viel später veröffentlichtes Forschungsreisetagebuch „Lachesis Lapponica or a tour in Lapland “ (1811).

LiNNÉs akademische Karriere als Nachfolger des ausscheidenden Professors RudBeck schien fast sicher, als ein ernst zu nehmender und erbittert gegen LinNÉ antretender Konkurrent von einem Auslandsaufenthalt zurückkehrte: der nur ein Jahr ältere promovierte Adjunkt der Medizin und später berühmte Kinderheilkundler Dr. Nils Rosenius (1706-1773; 1762 geadelt zu NiLs Rosén von Rosenstein), der ebenfalls auf die freiwerdende Professur Rudbecks spekulierte. Vermutlich war somit ein weiterer Grund für die Durchführung der Lapplandreise 


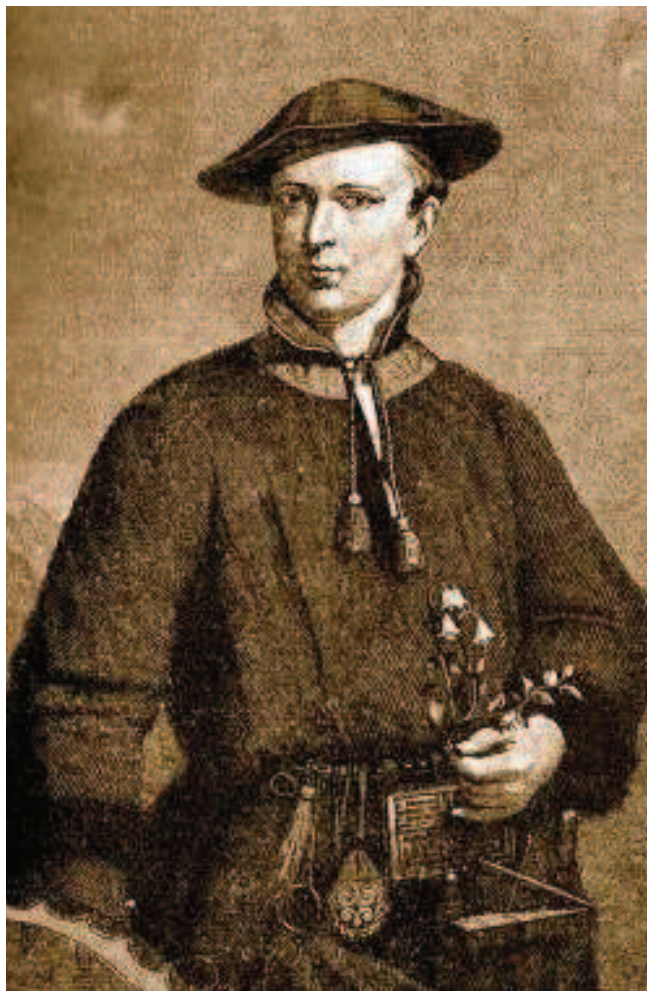

die Tatsache, dass LinNé sich dem sehr erfolgreichen RoséN in den Weg stellen und sich gleichfalls mit einer aufsehenerregenden Forschungsreise rühmen wollte. Auch die zweite Studienreise LinNÉs nach Dalarna im Jahre 1734 mag vor diesem Hintergrund zu sehen sein, diente aber in erster Linie der Vertiefung seiner Kenntnisse in Mineralogie und Bergbauwesen. LinNé lernte Weihnachten 1734 auf dieser Reise in der Bergbaustadt Falun Sara Elisabeth Moraea (1716-1806), die Tochter des dortigen Arztes, kennen. Bereits wenige Wochen später verlobte sich LinNé mit SARA LIsA. Eine zukünftige Heirat hingegen sollte auf Wunsch des Brautvaters erst nach drei Jahren stattfinden, wenn LinNé hoffentlich als Arzt tätig sein und über ein gesichertes Einkommen verfügen würde, um eine Familie ernähren zu können. Andererseits wird vermutet, dass der zukünftige Schwiegervater LinNÉ auch finanziell unter die Arme gegriffen hat, damit dieser sein Medizinstudium möglichst zügig beenden konnte.
Während seiner Forschungsaufenthalte und in den Monaten dazwischen wurden die Auseinandersetzungen zwischen RoséN und LINNÉ in Uppsala um die Nachfolge der RudBecK'schen Professur immer heftiger und endeten schließlich sogar zeitweilig mit einem Zerwürfnis zwischen RudBeck und LinNÉ. Ihm wurde klar, dass er zur erfolgreichen Fortführung seiner akademischen Laufbahn möglichst bald die Doktorwürde erlangen musste. $\mathrm{Zu}$ dieser Zeit war es üblich, zum Zwecke der Promotion an eine Universität des Auslandes zu wechseln, da damals an den schwedischen Universitäten aus Personal- und Geldmangel kaum noch Dissertationen abgenommen wurden. LINNÉ wählte hierzu die heute nicht mehr existierende Universität Harderwijk in Holland, die bei schwedischen Studenten beliebt war, da man dort offenbar schnell und kostengünstig den medizinischen Doktorgrad erlangen konnte. Im Sommer 1735 machte sich daher LiNNé nach Holland auf.

LINNÉ verband eine enge Freundschaft zu einem gleichaltrigen Mitstudenten, PeHr ARTEDI (1705-1735). Zusammen begannen sie ein System der Klassifizierung der Organismen zu erarbeiten, wobei sich ARTEDI besonders mit den Fischen auseinander setzte. Es steht außer Zweifel, dass viele Ideen zur Artdiagnose, Artbeschreibung und Nomenklatur, die LinNÉ später anwendete, zusammen mit ARTEDI entwickelt wurden. ARTEDI folgte LinNÉ nach Holland. Leider wurde diese fruchtbare Zusammenarbeit der beiden jungen Wissenschaftler durch den tragischen und frühen Unfalltod des jungen Zoologen in Amsterdam beendet. ARTEDIs begonnene Manuskripte vervollständigte und veröffentlichte LINNÉ jedoch später unter seinem eigenen Namen.

\subsection{Promotion und Auslandsaufenthalt}

Schon wenige Tage nach seiner Ankunft konnte LinNé in Harderwijk den gewünschten Doktortitel erwerben. Er legte dazu eine Disserta-

Abb. 8: LinNÉ in lappländischer Tracht, in der Hand ein Moosglöckchen. Portrait von M. Hoffman, ca. 1735. 


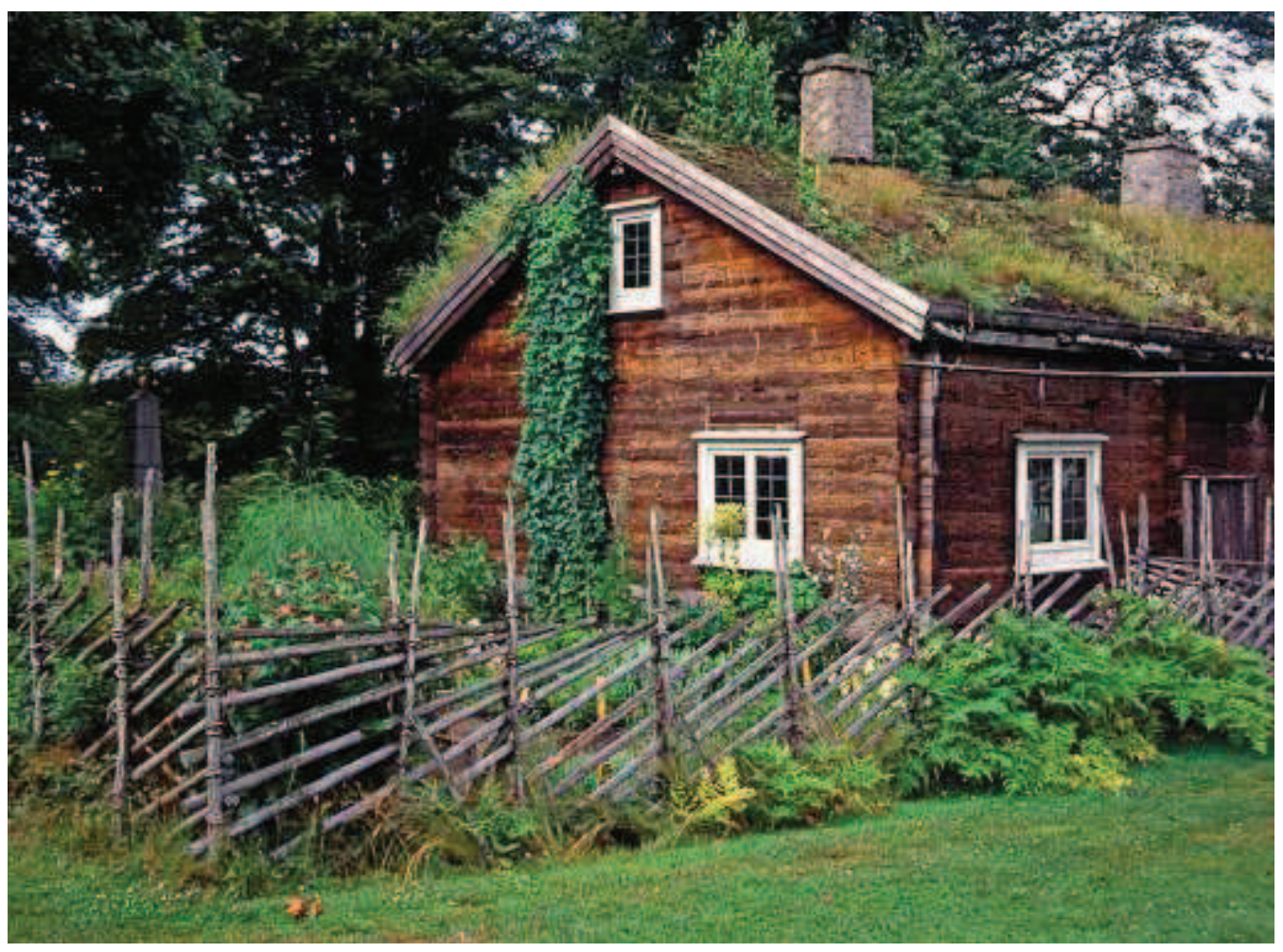

tionsschrift über Intermittierendes Fieber vor, die er bereits in Schweden verfasst hatte. So schnell zum Ziel gelangt, verließ LinNé die Kleinstadt Harderwijk und reiste sogleich zur renommierten Universität in Leiden weiter, um sich dort vorrübergehend seinen eigentlichen Forschungsinteressen, der Botanik und der Systematik der Pflanzenwelt, zu widmen. Auch in Holland hatte LinNÉ erneut Glück und fand schon kurz nach seinem Eintreffen Gönner, die ihn und seine Arbeit finanziell und ideell unterstützten. Diesmal waren es vor allem JAN FrEDERIK Gronovius (1686-1762) und der angesehene Medizinprofessor Herman BoerhaAve (1668-1738) aus Leiden sowie Johannes BuRMAN (1706-1779) in Amsterdam, in dessen Haus LINNÉ vorrübergehend wohnte und der ihn letztlich auch überredete, in Holland $\mathrm{zu}$ bleiben. Zwischen 1735 und 1738 lebte, arbeitete und publizierte LINNÉ an verschiedenen

Abb. 9: Råshult, das Geburtshaus von LinNé.
Orten in Holland und besuchte von dort aus auch Wissenschaftler und wissenschaftliche Einrichtungen in Dänemark, Deutschland, England und Frankreich. Auf Vorschlag von Gronovius und BoerhaAve wurde LinNÉ Leiter des großen botanischen und zoologischen Gartens in Hartekamp, der sich im Privatbesitz des wohlhabenden Kaufmanns George ClifFORD (1685-1760) befand. LINNÉ wohnte zwei Jahre in größerem Wohlstand als je zuvor auf dem Landsitz dieses reichen Gönners und konnte sich in dieser Position voll und ganz auf seine Arbeiten zur Systematik der Tier- und Pflanzenwelt konzentrieren. Nebenbei betreute er den an einer hypochondrischen Störung leidenden Clifford medizinisch und katalogisierte und erweiterte den Pflanzenbestand des „Hortus Cliffortianus“ (1737). Dabei gelang ihm erstmals in Europa, eine Bananenpflanze zum Blühen und Fruchten zu bringen, was zur damaligen Zeit große Bewunderung hervorrief. Bereits 1735 hatte LINNÉ - unter finanzieller Unterstützung von Gronovius - die erste Aus- 


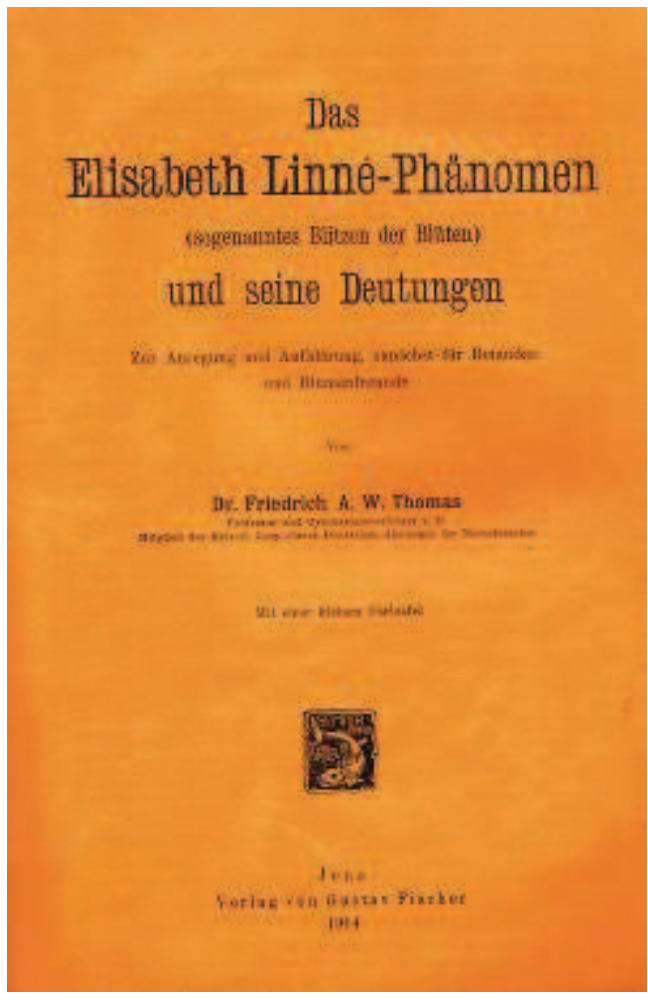

gabe seines wohl wichtigsten Werkes über Taxonomie, der „Systema Naturae“, veröffentlicht, das überwiegend sehr positiv und bewundernd aufgenommen wurde (s.u.). Allein im Jahr 1737 hatte LiNné in Holland schon insgesamt etwa 1850 Druckseiten veröffentlicht.

\subsection{Berufliches Wirken und Familiengrün- dung}

LinNÉ entschied sich im Frühjahr 1738 aus familiären Gründen für eine Heimkehr nach Schweden. Nach kurzen Zwischenstationen in Leiden, Paris und in Stenbrohult bei seinem Vater sowie in Falun im Hause seiner Verlobten siedelte sich LinNé in Stockholm an, in der Hoffnung, in der Hauptstadt möglichst schnell als Arzt Fuß zu fassen. Er eröffnete eine Arztpraxis und wurde bald ein erfolgreicher Stadtarzt. Zusätzlich wurde er als Admiralitätsmedicus an die Stockholmer Marinestation berufen, wo er für die ärztliche Betreuung schwedischer Marinesoldaten zuständig war. Neben seiner ärztlichen Tätigkeit verwendete LINNÉ in Stockholm aber weiterhin viel Zeit für seine botanischen und taxonomischen Studien. Im Mai 1739 trat LINNÉ als Mitbegründer der Königlichen Schwedischen Akademie der Wissenschaften in Stockholm auf, dessen erster Präsident er wurde. Wegen der beruflichen und wissenschaftlichen Erfolge stand 1793 somit der Heirat mit Sara Lisa Moraea nichts mehr im Wege.

Am 20. Januar 1741 wurde als erstes Kind des Paares der einzige überlebende Sohn CARL LINNÉ der Jüngere (1741-1783) geboren. Es folgten die fünf Töchter Elisabet CHristina (1743-1782), Sara Magdalena (1744-1744), Lovisa (1749-1839), SARA Christina (17511835), Sophia (1757-1830) und ein weiterer Sohn Johannes Linnaeus (1754-1757). Das Jahr der Geburt seines ältesten Sohnes markiert auch einen weiteren Einschnitt im Leben LINNÉs: Am 5. Mai 1741 wurde LinNÉ als Nachfolger von Lars Roberg zum Professor der theoretischen und praktischen Medizin an die Universität Uppsala berufen. Bei der Ausschreibung der Stelle und ihrer Besetzung mit Linné soll es einige heftige Auseinandersetzungen zwischen betroffenen Wissenschaftlern gegeben haben, die sich u. a. aus den alten Streitigkeiten zwischen Linné und Rosén, der ja mittlerweile den anderen medizinischen Lehrstuhl in Uppsala als Nachfolge von RudBEck inne hatte, ergaben. Später fand aber eine Versöhnung mit RosÉN statt, als die beiden Professoren 1742 ihre Lehrstühle tauschten. Von nun an lehrte Rosén Medizin und Anatomie, LINNÉ hingegen Botanik, allgemeine Naturgeschichte sowie Ernährungswissenschaften. Als wichtigster Gönner und Freund seiner Zeit als Professor in Uppsala (1741-1772) gilt Graf Carl Gustav Tessin (1695-1770) ein sehr naturwissenschaftlich interessierter und einflussreicher Politiker.

In den folgenden Jahrzehnten, in denen er auch mehrmals zum Rektor der Universität berufen wurde, führte LINNÉ einige weitere kleinere Forschungsreisen im eigenen Lande durch u. a. nach Gotland und Öland (1741) sowie in

Abb. 10: Abhandlung über das Elisabeth LinNÉ-Phänomen der blitzenden Blüten aus dem Jahr 1914. 
die Provinzen Västergötland (1746) und Schonen (1749). Daneben hatte er zahlreiche Schüler, die in vielen verschiedenen Ländern der Erde für ihn Forschungen durchführten und ihn stets mit unbekanntem Pflanzen- und Tiermaterial versorgten. Manche dieser Apostel, wie LINNÉ seine reisenden Schüler liebevoll selber nannte, verloren auf diesen gefährlichen Reisen sogar ihr Leben. Andere wurden selbst berühmte Professoren. Unter den bekanntesten seiner Schüler sind PeHr Kalm (1716-1779, schwedisch-finnischer Entdecker, Botaniker und Agrarökonom.), der u. a. auf seinen Nordamerikareisen die Niagara-Fälle entdeckte, PEHR FORSSKÅL (1732-1763, finnischer Naturkundler und Orientalist), Daniel Solander (17331782, schwedischer Botaniker in London), der Cook auf seiner ersten Weltumseglung begleitete, Paul Dietrich Giseke (I74I-I796, deutscher Arzt, Botaniker, Lehrer und Bibliothekar in Hamburg), Carl Peter Thunberg (17431828, schwedischer Naturwissenschaftler und Philosoph in Uppsala), Anders Sparrman (1748-1820, schwedischer Naturkundler), der Cook auf dessen zweiter Weltumseglung begleitete, Johann Christian Fabricius (I745I808, dänischer Zoologe und Wirtschaftswissenschaftler in Kiel) sowie ANDERs DAHL (1751-1789, Botaniker und Mediziner in Turku, Finnland).

Viel Zeit verwendete LINNÉ in Uppsala auch für die Neugestaltung und Erweiterung des Botanischen Gartens, dessen Leiter er war und in dem auch sein Wohnhaus stand. Dieser Garten wird heute auch als LinNé-Garten bezeichnet und enthält bis zum heutigen Tage noch einige Pflanzenindividuen, die Linné kultiviert oder gepflanzt hat. LinNé erweiterte zur brandgeschützten Unterbringung seiner umfangreichen Sammlungen von Pflanzen, Tieren und Mineralien noch im gleichen Jahr das 1758 als Sommerwohnsitz erworbene Gut Hammarby wenige Kilometer außerhalb von Uppsala, wo er schließlich 1769 auch ein Museum eröffnete.

Abb.11: Das Adelswappen der Familie v. Linné in einer unkolorierten Darstellung, verändert aus GoERKe (1989).

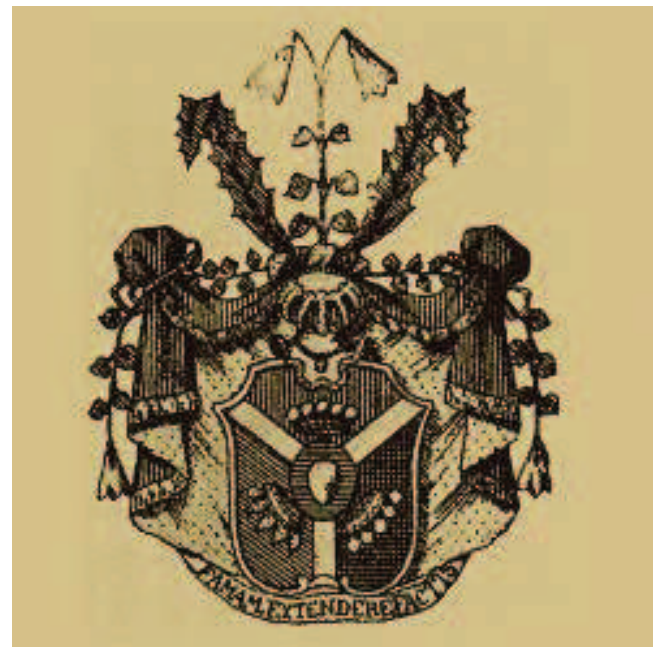

Höhepunkt seines Schaffens in Uppsala war u.a. die Herausgabe des Buches „Species plantarum" (1753), in dem LinNé die Grundsteine zur modernen botanischen Nomenklatur legte. Aufgrund seiner großen wissenschaftlichen Erfolge wurde LinNé 1761 zum Ritter geadelt. Er änderte daraufhin seinen Namen von CAROLUS LinNaEus in CARL vON LINNÉ und entwickelte ein Adelswappen (Abb. 11) der Familie Linné. Dieses zeigt außer der Pflanze Linnaea in späteren Fassungen in der Mitte drei verschieden gefärbte Felder, die für die drei von LINNÉ klassifizierten Bereiche der Natur (Gesteine, Tiere und Pflanzen) stehen.

\subsection{Lebensabend und Nachkommen}

LINNÉ, der schon immer häufig an verschiedenen Krankheiten wie heftigen Migräneanfällen, Fieberschüben und rheumatischen Leiden litt, trat aufgrund gesundheitlicher Probleme bereits 1763 von seinen Lehrtätigkeiten zurück. Diese übernahm für ihn sein Sohn CARL, der ebenfalls Medizin und Botanik in Uppsala studiert hatte, aber längst nicht die wissenschaftlichen Fähigkeiten und Interessen seines Vaters aufwies. Schon mit 18 Jahren war dieser unter dem Einfluss seines Vaters zum Demonstrator der Botanik ernannt worden, mit 23 Jahren erhielt er die Ehrendoktorwürde, ohne je eine Dissertation geschrieben zu haben, was unter den wissenschaftlichen Kollegen nicht unumstritten blieb. 


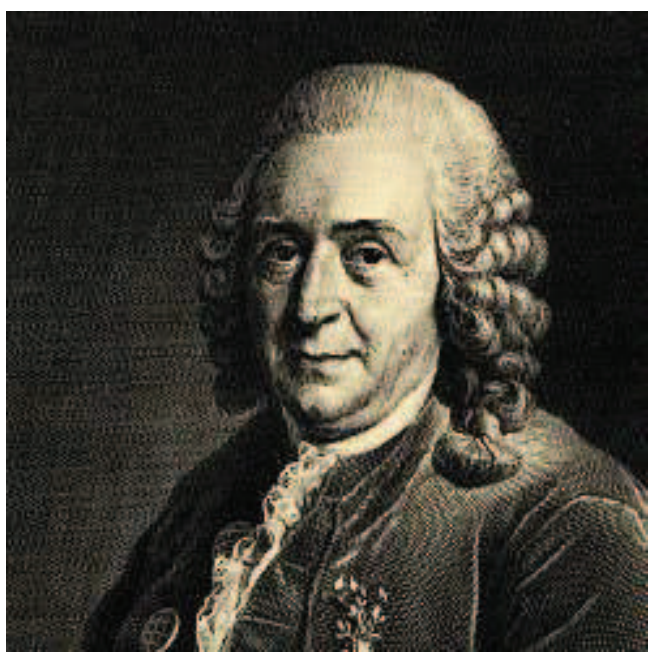

Bis 1772 beaufsichtigte LinNé die Arbeiten am Lehrstuhl des Sohnes selbst, danach wurde seine Schaffenskraft erheblich durch seinen schlechten Gesundheitszustand geschmälert. Während sich Linné und sein Kollege Rosén in den letzten Jahren ihrer nun intensiven Freundschaft bis zum Tode RosÉNs 1773 gegenseitig ärztlich betreut hatten, stand LiNNÉ nun kein guter befreundeter Arzt mehr zur Seite. 1774 hatte er einen Schlaganfall, ein zweiter folgte 1776, von dessen Folgen er sich nie wieder erholte. In seiner Autobiographie schreibt er selber über sich: „LinNÉ hinkt, kann kaum gehen, redet undeutlich, kann kaum schreiben."Bald darauf kam es auch zu einem raschen Verfall der geistigen Kräfte. In den letzten Monaten seines Lebens war Linné ein hilfloser Pflegefall. Er starb am 10. Januar 1778 und wurde am 23. Januar 1778 in der Domkirche von Uppsala in einem großen Festakt beigesetzt. LINNÉ hinterließ ein umfangreiches Werk wie kaum ein anderer zuvor. Sein Sohn überlebte ihn nur um fünf Jahre und starb unverheiratet. Der LINNÉ-Schüler CARL Peter Thunberg übernahm daraufhin Linnés Professur. Nach dem Tode des Sohnes verkaufte die Witwe LinNÉs aus Geldmangel sämtliche Sammlungen, Herbarien und Unterlagen ihres Mannes an einen Engländer namens Sir JAMES EDward SMith (1750-1828), was der Ausgangspunkt für die Gründung der LinneaN Society zu London war. Von LinNÉs Töchtern bekamen nur die älteste, Elisabeth Christina, und die beiden jüngsten Töchter, Sara ChrisTINa und Sophia, Kinder, die aber natürlich nicht den Namen v. Linné trugen. Elisabeth Christina heiratete Carl Frederik BergenCRANTZ (1726-1792), den Enkel des Medizinprofessors Olaf Rudbeck. Sie ist die einzige der übrigen Kinder LinNÉs, die sich mit Botanik beschäftigte und 1762 an der schwedischen Akademie der Wissenschaften über die von ihr gemachte Entdeckung des Leuchtens der Blüte der Kapuzinerkresse berichtete (Abb. 10). Heute treffen sich in Schweden regelmäßig noch etwa 200 Nachkommen LinNÉs, die sich ihres berühmten Ahnen erinnern.

\section{Literatur}

Glässer. E., Lindemann, R. \& Venzke, J.-F. 2003:

Nordeuropa. - Darmstadt.

Goerke, H. 1989: Carl von Linné. Arzt, Naturforscher, Systematiker. - Stuttgart.

Korneck, D., Schnittler, M. \& Vollmer, I. 1996: Rote Liste der Farn- und Blütenpflanzen (Pteridophyta et Spermatophyta) Deutschlands. - Schr.-R. f.

Vegetationskunde 28: 21-187.

Mossberg, B., Sternierg, L. \& Ericsson, S. 1992: Den Nordiska Floran. - Solna.

\section{Internetseiten}

http://www.univie.ac.at/science-

archives/wissenschaftstheorie_2/: OESER, E. 2003:

System, Klassifikation, Evolution. - Wien.

http://www.biologie.uni-hamburg.de/b-

online/d01/linne.htm: v. SEnGBUSCH, P. 2000:

Botanik online - die Internetlehre:

Stichwort CARL v. LinNÉ, Universität Hamburg,

Abb.12: LinNé 1775, Kupferstich nach einem Gemälde von Alexander Roslin, verändert aus Goerke (1989). 